\title{
Management of chronic hepatitis B before and after liver transplantation
}

\author{
B Wang, K Agarwal, D Joshi
}

- Additional material is published online only. To view please visit the journal online (http://dx.doi.org/10.1136/ flgastro-2016-100768).

Institute of Liver Studies, King's College Hospital NHS Foundation Trust, London, UK

\section{Correspondence to}

Dr Bo Wang, Institute of Liver

Studies, King's College Hospital, Denmark Hill, London SE5 9RS, UK; Bo.wang@nhs.net

Received 01 November 2016 Revised 19 January 2017

Accepted 22 January 2017 Published Online First 14 February 2017

\section{CrossMark}

To cite: Wang $B$,

Agarwal K, Joshi D.

Frontline Gastroenterology

2018;9:79-84.

\begin{abstract}
Chronic hepatitis B infection is a global public health problem associated with significant morbidity and mortality. Persistent infection may evolve to liver cirrhosis and hepatocellular carcinoma, and hepatitis B-related liver disease is a common indication for liver transplantation. Patients with advanced liver disease should be treated with antiviral therapy which may result in clinical improvement. The management of patients after liver transplant then focuses on preventing hepatitis B recurrence in the graft. With the introduction of prophylactic treatment, patient and graft survival has improved significantly. In this review, we will discuss the management of patients with hepatitis B-related cirrhosis, both compensated and decompensated. We also review the management of hepatitis B after liver transplantation.
\end{abstract}

\section{INTRODUCTION}

Chronic infection with hepatitis $\mathrm{B}$ virus (HBV) affects an estimated 350-400 million people worldwide with prevalence as high as $5 \%-10 \%$ of the adult population in Sub-Saharan Africa and East Asia; in Europe over 13 million people live with HBV infection, a prevalence of nearly $2 \% .{ }^{1}$ Persistent chronic hepatitis B may evolve to cirrhosis and hepatocellular carcinoma (HCC) and deaths attributed to these complications are estimated at 780000 per year. ${ }^{2}$ In patients with HBV-related cirrhosis, the 5-year cumulative incidence of hepatic decompensation is around $20 \%$. Without transplantation, the 5 -year survival of these decompensated patients is $15 \%-35 \%$.

Current management of chronic hepatitis $\mathrm{B}$ includes pharmacological agents such as interferon and nucleos(t)ide analogues (NA), as well as surveillance for HCC and management of decompensation. $^{3}$ Despite this however, liver transplantation may still be necessary in those patients who develop indications with decompensated cirrhosis, acute hepatic flare or HCC. Appropriate management of patients with HBV-related cirrhosis is therefore key in either preventing progression to transplant, or to ensuring optimisation going into transplant. After liver transplantation, the patient remains at risk of persistent HBV infection and prevention of recurrent infection in the graft is of utmost importance in ensuring graft and patient survival. In this article, we aim to review the management of chronic hepatitis B before transplantation, in patients with compensated and decompensated cirrhosis and also the management after liver transplantation.

\section{MANAGEMENT BEFORE LIVER TRANSPLANTATION}

\section{Treatment in compensated cirrhosis}

Patients with previously undiagnosed and untreated hepatitis B infection presenting with cirrhotic liver disease is not an uncommon scenario. After confirmation of cirrhosis on the basis of clinical, pathological and radiological assessment, a systematic screen should be undertaken to elicit the underlying cause. Patients identified as having chronic HBV infection with hepatitis B surface antigen (HBsAg) positivity should be checked for cofactors such as alcoholic or non-alcoholic fatty liver disease and other viral co-infection. Once the diagnosis of chronic hepatitis B-related cirrhosis has been made, the consensus is all of these patients should be treated with antiviral therapy including those with decompensation. ${ }^{34}$ This is in contrast to patients presenting before the development of cirrhosis where the decision to treat is based on hepatitis $\mathrm{B}$ e antigen status, alanine transaminase 
derangement, HBV DNA level and the degree of liver fibrosis either by histological assessment or by noninvasive methods such as transient elastography. ${ }^{3}$

The rationale of commencing treatment in those with compensated HBV-related cirrhosis is to prevent further deterioration of liver function or progression to decompensation, as well as reducing the risk of development of HCC. Liaw et $a l,{ }^{5}$ in a landmark randomised controlled trial of patients with biopsyproven advanced fibrosis or cirrhosis, compared untreated patients with those treated with lamivudine and showed significantly fewer events in the treatment group such as deterioration in Child-Pugh score, spontaneous bacterial peritonitis, portal hypertensive bleeding, development of HCC and death related to liver disease. Further studies have consolidated this and shown significantly improved longterm survival in patients with cirrhosis with sustained HBV DNA suppression on antiviral therapy. ${ }^{6}$ Furthermore, there is evidence that prolonged treatment and viral suppression result in improvement histologically with regression of advanced fibrosis and even $^{\text {cirrhosis. }}{ }^{78}$

The risk of developing HCC in patients with HBV-related cirrhosis is estimated at 10\%-17\% cumulatively over 5 years. ${ }^{9}$ Risk factors can be divided into host factors and virus factors. Host factors include male sex, age and family history of HCC. The virus factors include HBV genotype, co-infection and duration of infection, but the most crucial remains HBV DNA level. A direct relationship between persistent elevation of HBV DNA ( $>10^{4}$ copies $/ \mathrm{mL}$ ) and risk of HCC was demonstrated by the Risk Evaluation of Viral load Elevation and Associated Liver disease/cancer Hepatitis B Virus (REVEAL-HBV) study group. ${ }^{10} 11$ Several studies and meta-analyses have gone on to demonstrate that successful viral suppression with antiviral therapy reduces the risk of HCC, particularly in patients with cirrhosis. ${ }^{12} 13$ The risk is not eliminated however and patients with cirrhosis on antiviral treatment should continue to have surveillance for HCC. ${ }^{14}$

\section{Treatment in decompensated cirrhosis and on transplant wait list}

Good evidence exists for treating patients with decompensated cirrhosis with antiviral therapy, with both short-term and long-term survival benefits. ${ }^{15} 16$ A recent large prospective study looked at outcomes in over 700 patients with decompensated HBV-related cirrhosis, of whom over 400 were treated with antiviral therapy in the form of various NA. ${ }^{17}$ This study demonstrated that antiviral treatment was associated with improved hepatic function (reflected as improvement in Child-Pugh and Model for End-stage Liver Disease (MELD) scores) and transplant-free survival. The study also showed long-term benefit in survival and improvement in liver function with a protracted follow-up period of $>5$ years. An earlier study in
2002 similarly demonstrated improved clinical and laboratory parameters compared with baseline in 154 patients with decompensated chronic hepatitis B treated with lamivudine for a median of 16 months. ${ }^{18}$ In both studies, a cohort of patients died in the first 6 months of follow-up despite antiviral therapy $\left(13.4 \%{ }^{17}\right.$ and $\left.16.2 \%{ }^{18}\right)$. It therefore suggests that some patients with very advanced disease cannot be salvaged with antiviral therapy and therefore would benefit from early assessment for liver transplantation. In the earlier study, predictors of 6-month mortality were found to be higher pretreatment serum bilirubin and creatinine, and detectable HBV DNA level at baseline. In practice, such patients should potentially undergo liver transplant assessment alongside commencing antiviral therapy. Clinical improvement with antiviral treatment on the wait list would then result in delisting.

In those patients for whom liver transplantation becomes necessary, viral suppression ideally to undetectable levels remains important in preventing HBV recurrence in the graft. Studies have shown a direct correlation between viral load at time of transplant and rate of recurrence. ${ }^{19}$ This, in conjunction with post-transplant prophylaxis, which will be discussed later in this review, has resulted in HBV recurrence rates falling from $70 \%$ to $100 \%$ to $<10 \% .^{20}$

\section{Therapeutic options}

The drugs currently approved for the treatment of chronic HBV include pegylated-interferon and five NA: lamivudine, adefovir dipivoxil, entecavir, telbivudine and tenofovir disoproxil fumarate (TDF). Interferon is not recommended in the treatment of patients with advanced cirrhosis given the increased risk of infection and hepatic decompensation. ${ }^{3}$ Of the NA, lamivudine has the longest history of evidence as the first oral agent. Early studies demonstrating the benefit of treating patients with cirrhosis with antiviral therapy used lamivudine and the same principles can be applied to other NA. However, with the development of newer NA, lamivudine is no longer recommended as first-line therapy because of its high rate of resistance over long-term use and its inferiority in terms of antiviral potency. ${ }^{3}{ }^{21}$ Viral breakthrough due to drug resistance can result in acute hepatic flare, which in patients with cirrhosis may potentially result in decompensation or even a more fulminant picture of hepatic failure. Entecavir and TDF are now the preferred first-line agents, with large systematic reviews demonstrating their efficacy in patients with both compensated and decompensated cirrhosis. ${ }^{16}{ }^{22}$ Both drugs are potent suppressors of HBV DNA with low rates of resistance; around $1 \%$ over 5 years of treatment for entecavir, ${ }^{23}$ and no reported resistance in long-term follow-up studies of TDF. ${ }^{84}$

The safety of drugs used in patients with cirrhosis is also a key issue as they lack hepatic reserve and may 
have co-existing problems such as renal impairment, which mean any adverse effects may be more detrimental. The safety of both entecavir and TDF has been evaluated in large randomised multicentre trials, which show no greater adverse events when compared with each other and with other older NA such as adefovir. ${ }^{25}{ }^{26}$ Nonetheless, there are theoretical concerns with both entecavir and TDF which require awareness. An association between entecavir and lactic acidosis has been suggested, with one report in which 5 out of 16 patients with decompensated cirrhosis developed severe lactic acidosis after starting entecavir treatment. ${ }^{27}$ TDF has been associated with reduced bone mineral density as well as with renal disease, from an asymptomatic rise in serum creatinine through to severe renal tubular disease manifest as Fanconi's syndrome. ${ }^{28}$

Tenofovir alafenamide (TAF) is a new drug which has been introduced as a potentially safer NA in longterm use. It is a prodrug to tenofovir which results in higher intracellular concentrations of the active metabolite, with lower circulating plasma levels which are associated with the aforementioned renal and bone toxicity. ${ }^{29}$ Two recent large phase III trials have demonstrated non-inferiority of TAF compared with TDF in terms of HBV DNA suppression at 48 weeks, as well as improved safety profile with respect to bone mineral density. ${ }^{30} 31$ These studies did not include patients with advanced liver disease (Childs-Pugh B or above) or decompensated cirrhosis; studies including these cohorts, as well as longer follow-up data, are planned.

\section{MANAGEMENT AFTER LIVER TRANSPLANTATION}

Recurrence of HBV infection after liver transplantation, defined as the reappearance of circulating HBsAg with or without detectable HBV DNA, is almost universal without appropriate management. Prior to the use of prophylaxis against HBV recurrence, graft and patient survival were so poor that $\mathrm{HBV}$-related liver disease was considered a relative if not absolute contraindication to transplantation. ${ }^{32}$ Passive prophylaxis with hepatitis B immunoglobulin (HBIG) reduced recurrence rates from $75 \%$ to $36 \%$, with improved graft and patient survival. ${ }^{33}$ After the development of oral NA antivirals, the options for prophylactic treatment have expanded.

\section{HBIG monotherapy}

The early studies with HBIG monotherapy used an intravenous dose intra-operatively during the anhepatic phase followed by daily dosing then subsequent monthly dosing aiming to maintain anti-HB titres of $>500 \mathrm{IU} / \mathrm{L}^{34}{ }^{35}$ Although short-term results were good, the need for indefinite therapy resulted in problems with local and systemic side effects, high costs and the development of HBIG-resistant mutants, which made it a poor long-term option. ${ }^{36}$

\section{NA monotherapy}

Early studies showed lamivudine and adefovir to be effective monotherapy in reducing HBV recurrence post-transplant. ${ }^{37}{ }^{38}$ However, as in the pretransplant setting, their long-term use is limited by high rates of resistance. More recently, entecavir monotherapy has been used with one study showing $77.5 \%$ of patients remained HBsAg negative 2 years after transplant. ${ }^{39}$ In this study, only $26 \%$ of patients were HBV DNA negative at the time of transplant. The same group went on to expand on this work by reviewing over 300 patients treated without HBIG prophylaxis, using NAs as monotherapy or in combination (predominantly lamivudine and adefovir). ${ }^{40}$ After 3 years, HBV recurrence rates ranged from $0 \%$ to $17 \%$ with 8 -year patient survival at $83 \%$. These results are promising, suggesting a role for limiting HBIG use and reducing financial costs.

\section{Combination therapy}

Combination therapy with HBIG and an NA has a large body of evidence, and is currently the recommended treatment regimen. ${ }^{3}$ Several large meta-analyses have demonstrated the efficacy of combination therapy, including reduced HBV-related mortality compared with HBIG alone. ${ }^{36}{ }^{41-43}$ More recently, there has been data showing even lower rates of recurrence using HBIG with a potent NA. An analysis including 11 trials (304 patients) of HBIG with either tenofovir or entecavir showed an overall 1\% recurrence rate. ${ }^{44}$ The specific HBIG regimen used in combination varies between centres but typically consists of an intravenous dose, usually 5000-10 000 IU, in the anhepatic phase and then immediately posttransplant, for example, $5000 \mathrm{IU} /$ day on alternate days for 1 week (figure 1). This is followed by intermittent low doses either at fixed intervals (monthly) or in a frequency dictated by hepatitis B surface antibody levels (usually to maintain levels $>50-100 \mathrm{IU} / \mathrm{L}$ ). ${ }^{45}$ Alternative routes of HBIG administration such as intramuscular or subcutaneous low doses are accepted strategies to reduce cost and adverse effects. ${ }^{46} 47$

Given the expense of long-term administration of HBIG, there has been recent interest in HBIG-free prophylaxis, with combination therapy for the immediate post-transplant period only and subsequent HBIG withdrawal. Single-centre studies have shown this to be safe and efficacious in patients at low risk of HBV recurrence. One group selected 47 patients treated with combination therapy who were considered low risk (mainly those with undetectable HBV DNA at transplant), to stop HBIG after 12 months. After HBIG discontinuation, patients were treated either with lamivudine plus adefovir or tenofovir, or tenofovir or entecavir monotherapy; $6.3 \%$ of patients developed HBsAg recurrence after a median follow-up of 24 months. ${ }^{48}$ Similarly, another group demonstrated $8.6 \%$ recurrence 22 months after HBIG 


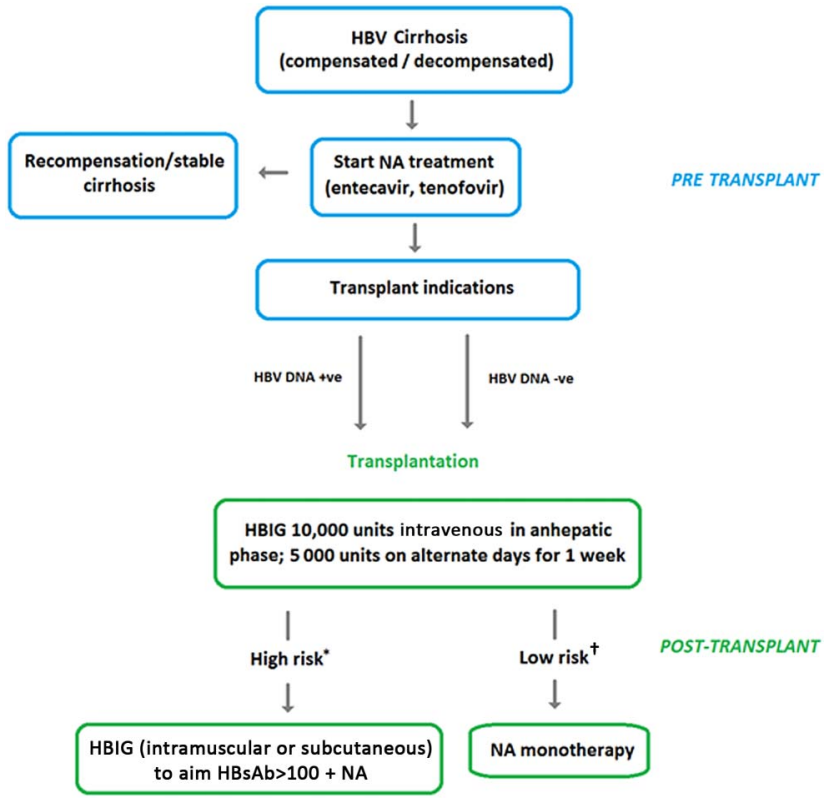

Figure 1 Potential treatment algorithm for management of HBV before and after liver transplantation (*HBV DNA positive, high-risk hepatocellular carcinoma recurrence, HBV/HDV co-infection; HHBV DNA negative). HBV, hepatitis B virus; NA, nucleos(t)ide analogue; HBsAg, hepatitis B surface antigen; HBIG, hepatitis B immunoglobulin; HDV, hepatitis D virus.

discontinuation in 58 patients treated with either tenofovir or entecavir after withdrawal. ${ }^{49}$

HBIG withdrawal, although potentially safe and efficacious, is only appropriate in selected patients. HBV DNA level at the time of transplant is the most important predictor of risk of recurrence and HBIG withdrawal should not be considered in those who are HBV DNA positive at the time of transplant. ${ }^{50}$ Other patients in whom HBIG-free prophylaxis would be inappropriate would be those transplanted for HCC at risk of recurrence, and those with limited treatment options if recurrence did occur, such as patients co-infected with hepatitis delta virus (HDV). HDV is the most aggressive form of viral hepatitis without any effective treatment options. ${ }^{51}$ Those transplanted with co-infection must remain $\mathrm{HBsAg}$ negative posttransplant to avoid recurrent delta hepatitis. With careful patient selection, HBIG-free prophylaxis is likely to become a standard part of post-transplant management of hepatitis B. A potential treatment algorithm could individualise management based on a patient's risk profile for HBV recurrence and identify patients in whom HBIG withdrawal would be appropriate (figure 1).

\section{CONCLUSION}

The availability of safe and effective antiviral therapy for the treatment of chronic hepatitis B has changed how we care for patients with advanced hepatitis B-related liver disease. With the ability to treat even those with decompensated cirrhosis effectively with minimal risk of resistance and few toxicities, improved survival with or without transplantation has become possible. For those who still require liver transplantation, the improvement in graft and patient survival with effective prophylactic treatment against viral recurrence has meant that transplantation is indeed an option. Future research will now look to novel therapies for a functional cure of hepatitis $\mathrm{B}$, which will impact on management before and after liver transplantation.

Contributors BW performed the literature review and wrote the article; KA and DJ provided editorial advice and input.

Competing interests None declared.

Provenance and peer review Not commissioned; externally peer reviewed.

\section{REFERENCES}

1 Hepatitis B. World Health Organisation fact sheet number 204, July 2015. http://www.who.int

2 Fattovich G, Zagni I, Scattolini C. Natural history of hepatitis B and prognostic factors of disease progression. Semin Liver Dis 2003;23:47-58.

3 European Association for the Study of the Liver. EASL clinical practice guidelines: management of chronic hepatitis B virus infection. J Hepatol 2012;57:167-85.

4 Terrault NA, Bzowej NH, Chang KM, et al. AASLD guidelines for treatment of chronic hepatitis B. Hepatology 2016;63:261-83.

5 Liaw YF, Sung JJY, Chow WC, et al. Lamivudine for patients with chronic hepatitis B and advanced liver disease. N Engl J Med 2004;351:1521-31.

6 Papatheodoridis GV, Dimou E, Dimakopoulos K, et al. Outcome of hepatitis B e antigen-negative chronic hepatitis B on long-term nucleos(t)ide analog therapy starting with lamivudine. Hepatology 2005;42:121-9. 
7 Chang TT, Liaw YF, Wu SS, et al. Long-term entecavir therapy results in the reversal of fibrosis/cirrhosis and continued histological improvement in patients with chronic hepatitis B. Hepatology 2010;52:886-93.

8 Marcellin P, Gane E, Buti M, et al. Regression of cirrhosis during treatment with tenofovir disoproxil fumarate for chronic hepatitis B: a 5-year open-label follow-up study. Lancet 2013;381:468-75.

9 Fattovich G, Bortolotti F, Donato F. Natural history of chronic hepatitis B: special emphasis on disease progression and prognostic factors. J Hepatol 2008;48:335-52.

10 Chen CJ, Yang HI, Su J, et al. Risk of hepatocellular carcinoma across a biological gradient of serum hepatitis B virus DNA level. JAMA 2006;295:65-73.

11 Chen CF, Lee WC, Yang HI, et al. Changes in serum levels of HBV DNA and alanine aminotransferase determine risk for hepatocellular carcinoma. Gastroenterology 2011;141:1240-8.

12 Baran B. Nucleos $(t)$ ide analogs in the prevention of hepatitis B virus related hepatocellular carcinoma. World J Hepatol 2015;7:1742-54.

13 Papatheodoridis GV, Lampertico P, Manolakopoulos S, et al. Incidence of hepatocellular carcinoma in chronic hepatitis B patients receiving nucleos(t)ide therapy: a systematic review. J Hepatol 2010;53:348-56.

14 Papatheodoridis GV, Manolakopoulos S, Touloumi G, et al. Virological suppression does not prevent the development of hepatocellular carcinoma in HBeAg-negative chronic hepatitis B patients with cirrhosis receiving oral antiviral(s) starting with lamivudine monotherapy: results of the nationwide HEPNET. Greece cohort study. Gut 2011;60:1109-16.

15 Shim JH, Lee HC, Kim KM, et al. Efficacy of entecavir in treatment-naïve patients with hepatitis B virus-related decompensated cirrhosis. J Hepatol 2010;52:176-82.

16 Singal AK, Fontana RJ. Meta-analysis: oral anti-viral agents in adults with decompensated hepatitis B virus cirrhosis. Alimen Pharmacol Ther 2012;35:674-89.

17 Jang JW, Choi JY, Kim YS, et al. Long-term effect of antiviral therapy on disease course after decompensation in patients with hepatitis B virus-related cirrhosis. Hepatology 2015;61:1809-20.

18 Fontana RJ, Hann HW, Perrillo RP, et al. Determinants of early mortality in patients with decompensated chronic hepatitis $\mathrm{B}$ treated with antiviral therapy. Gastroenterology 2002;123:719-27.

19 Marzano A, Gaia S, Ghisetti V, et al. Viral load at the time of liver transplantation and risk of hepatitis B virus recurrence. Liver Transpl 2005;11:402-9.

20 Manne V, Allen RM, Saab S. Strategies for the prevention of recurrent hepatitis B virus infection after liver transplantation. Gastroenterol Hepatol 2014;10:175-9.

21 Peng CY, Chien RN, Liaw YF. Hepatitis B virus-related decompensated liver cirrhosis: benefits of antiviral therapy. J Hepatol 2012;57:442-50.

22 Lok AS, McMahon BJ, Brown RS, et al. Antiviral therapy for chronic hepatitis B viral infection in adults: a systematic review and meta-analysis. Hepatology 2016;63:284-306.

23 Fontana RJ. Entecavir in decompensated HBV cirrhosis: the future is looking brighter. J Hepatol 2010;52:147-9.

24 Kitrinos KM, Corsa A, Liu Y, et al. No detectable resistance to tenofovir disoproxil fumarate after 6 years of therapy in patients with chronic hepatitis B. Hepatology 2014;59:434-42.

25 Liaw YF, Raptopoulou-Gigi M, Cheinquer H, et al. Efficacy and safety of entecavir versus adefovir in chronic hepatitis B patients with hepatic decompensation: a randomized, open-label study. Hepatology 2011;54:91-100.

26 Liaw YF, Sheen IS, Lee CM, et al. Tenofovir disoproxil fumarate (TDF), emtricitabine/TDF, and entecavir in patients with decompensated chronic hepatitis B liver disease. Hepatology 2011;53:62-72.

27 Lange CM, Bojunga J, Hofmann WP, et al. Severe lactic acidosis during treatment of chronic hepatitis B with entecavir in patients with impaired liver function. Hepatology 2009;50:2001-6.

28 Woodward CLN, Hall AM, Williams IG, et al. Tenofovir-associated renal and bone toxicity. HIV Med 2009;10:482-7.

29 Agarwal K, Fung SK, Nguyen TT, et al. Twenty-eight day safety, antiviral activity, and pharmacokinetics of tenofovir alafenamide for treatment of chronic hepatitis B infection. J Hepatol 2015;62:533-40.

30 Buti M, Gane E, Seto WK, et al. Tenofovir alafenamide versus tenofovir disoproxil fumarate for the treatment of patients with $\mathrm{HBeAg}$-negative chronic hepatitis B virus infection: a randomised, double-blind, phase 3, non-inferiority trial. Lancet Gastroenterol Hepatol 2016;1:196-206.

31 Chan HLY, Fung S, Seto WK, et al. Tenofovir alafenamide versus tenofovir disoproxil fumarate for the treatment of HBeAg-positive chronic hepatitis B virus infection: a randomised, double-blind, phase 3, non-inferiority trial. Lancet Gastroenterol Hepatol 2016;1:185-95.

32 Eason JD, Freeman RB Jr, Rohrer RJ, et al. Should liver transplantation be performed for patients with hepatitis $\mathrm{B}$ ? Transplantation 1994;57:1588-93.

33 Samuel D, Muller R, Alexander G, et al. Liver transplantation in European patients with the hepatitis B surface antigen. N Engl J Med 1993;329:1842-7.

34 Terrault NA, Zhou S, Combs C, et al. Prophylaxis in liver transplant recipients using a fixed dosing schedule of hepatitis B immunoglobulin. Hepatology 1996;24:1327-33.

35 Sawyer RG, McGory RW, Gaffey MJ, et al. Improved clinical outcomes with liver transplantation for hepatitis B-induced chronic liver failure using passive immunization. Ann Surg 1998;227:841-50.

36 Loomba R, Rowley AK, Wesley R, et al. Hepatitis B immunoglobulin and lamivudine improve hepatitis B-related outcomes after liver transplantation: meta-analysis. Clin Gastro Hер 2008;6:696-700.

37 Perrillo RP, Wright T, Rakela J, et al. A multicentre United States-Canadian trial to assess lamivudine monotherapy before and after liver transplantation for chronic hepatitis B. Hepatology 2001;33:424-32.

38 Schiff E, Lai CL, Hadziyannis S, et al. Adefovir dipivoxil for wait-listed and post-liver transplantation patients with lamivudine-resistant hepatitis B: final long-term results. Liver Transpl 2007;13:349-60.

39 Fung J, Cheung C, Chan SC, et al. Entecavir monotherapy is effective in suppressing hepatitis B virus after liver transplantation. Gastroenterology 2011;141:1212-19.

40 Fung J, Chan SC, Cheung C, et al. Oral nucleoside/nucleotide analogs without hepatitis B immune globulin after liver transplantation for hepatitis B. Am J Gastroenterol 2013;108:942-8.

41 Rao W, Wu X, Xiu D, et al. Lamivudine or lamivudine combined with hepatitis B immunoglobulin in prophylaxis of hepatitis B recurrence after liver transplantation: a meta-analysis. Transpl Int 2009;22:387-94. 
42 Cholongitas E, Goulis J, Akriviadis E, et al. Hepatitis B immunoglobulin and/or nucleos(t)ide analogues for prophylaxis against hepatitis $b$ virus recurrence after liver transplantation:a systematic review. Liver Transpl 2011;17:1176-90.

43 Katz LH, Paul M, Gay DG, et al. Prevention of recurrent hepatitis B virus infection after liver transplantation: hepatitis B immunoglobulin, antiviral drugs, or both? Systematic review and meta-analysis. Transpl Infect Dis 2010;12:292-308.

44 Cholongitas E, Papatheodoridis GV. High genetic barrier nucleos $(\mathrm{t})$ ide analogue(s) for prophylaxis from hepatitis B virus recurrence after liver transplantation: a systematic review. Am J Transpl 2013;13:353-62.

45 Jiang L, Yan L, Li B, et al. Prophylaxis against hepatitis B recurrence posttransplantation using lamivudine and individualised low-dose hepatitis B immunoglobulin. Am J Transplant 2010;10:1861-9.

46 De Simone P, Romagnoli R, Tandoi F, et al. Early introduction of subcutaneous hepatitis $\mathrm{B}$ immunoglobulin following liver transplantation for hepatitis B virus infection: a prospective, multicentre study. Transplantation 2016;100:1507-12.

47 Gane EJ, Angus PW, Strasser S, et al. Lamivudine plus low-dose hepatitis B immunoglobulin to prevent recurrent hepatitis B following liver transplantation. Gastroenterology 2007;132:931-7.

48 Cholongitas E, Vasiliadis T, Antoniadis N, et al. Hepatitis B prophylaxis post liver transplantation with newer nucleos $(\mathrm{t})$ ide analogues after hepatitis B immunoglobulin discontinuation. Transpl Infect Dis 2012;14:479-87.

49 Fernández I, Loinaz C, Hernández O, et al. Tenofovir/entecavir monotherapy after hepatitis $\mathrm{B}$ immunoglobulin withdrawal is safe and effective in the prevention of hepatitis B in liver transplant recipients. Transplant Infect Dis 2015;17:695-701.

50 Fox AN, Terrault NA. The option of HBIG-free prophylaxis against recurrent HBV. J Hepatol 2012;56:1189-97.

51 Wedemeyer H, Manns MP. Epidemiology, pathogenesis and management of hepatitis D: update and challenges ahead. Nat Rev Gastroenterol Hepatol 2010;7:31-40. 